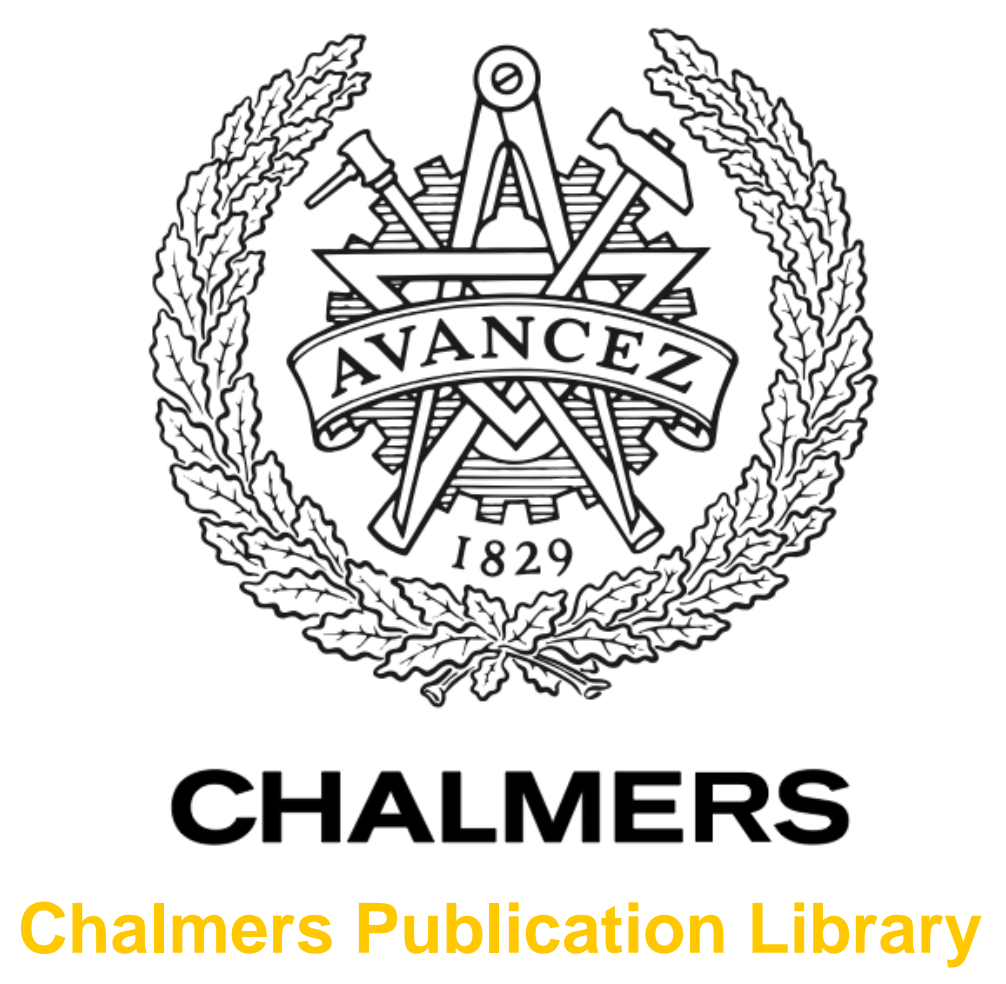

\author{
Optimal Energy Beamforming and Data Routing for Immortal Wireless Sensor \\ Networks
}

This document has been downloaded from Chalmers Publication Library (CPL). It is the author's version of a work that was accepted for publication in:

IEEE International Conference on Communications (ICC) (2017)

Citation for the published paper:

Du, R. ; Ozcelikkale, A. ; Fischione, C. et al. (2017) "Optimal Energy Beamforming and Data Routing for Immortal Wireless Sensor Networks". IEEE International Conference on Communications (ICC) (2017)

Downloaded from: http://publications.lib.chalmers.se/publication/248105

Notice: Changes introduced as a result of publishing processes such as copy-editing and formatting may not be reflected in this document. For a definitive version of this work, please refer to the published source. Please note that access to the published version might require a subscription.

Chalmers Publication Library (CPL) offers the possibility of retrieving research publications produced at Chalmers University of Technology. It covers all types of publications: articles, dissertations, licentiate theses, masters theses, conference papers, reports etc. Since 2006 it is the official tool for Chalmers official publication statistics. To ensure that Chalmers research results are disseminated as widely as possible, an Open Access Policy has been adopted.

The CPL service is administrated and maintained by Chalmers Library. 


\title{
Optimal Energy Beamforming and Data Routing for Immortal Wireless Sensor Networks
}

\author{
Rong Du*, Ayca Ozcelikkale ${ }^{\ddagger}$, Carlo Fischione*, Ming Xiao ${ }^{\dagger}$ \\ * Automatic Control Department, ${ }^{\ddagger}$ Department of Signals and Systems, ${ }^{\dagger}$ Communication Theory Department \\ ${ }^{*},{ }^{\dagger}$ KTH Royal Institute of Technology, ${ }^{\ddagger}$ Chalmers University of Technology, Sweden \\ Email: \{rongd, carlofi, mingx\}@kth.se, ayca.ozcelikkale@chalmers.se
}

\begin{abstract}
Wireless sensor networks (WSNs) consist of energy limited sensor nodes, which limits the network lifetime. Such a lifetime can be prolonged by employing the emerging technology of wireless energy transfer (WET). In WET systems, the sensor nodes can harvest wireless energy from wireless charger, which can use energy beamforming to improve the efficiency. In this paper, a scenario where dedicated wireless chargers with multiple antennas use energy beamforming to charge sensor nodes is considered. The energy beamforming is coupled with the energy consumption of sensor nodes in terms of data routing, which is one novelty of the paper. The energy beamforming and the data routing are jointly optimized by a non-convex optimization problem. This problem is transformed into a semidefinite optimization problem, for which strong duality is proved, and thus the optimal solution exists. It is shown that the optimal solution of the semi-definite programming problem allows to derive the optimal solution of the original problem. The analytical and numerical results show that optimal energy beamforming gives two times better monitoring performance than that of WET without using energy beamforming.
\end{abstract}

\section{INTRODUCTION}

Wireless sensor networks (WSNs) have been widely used to monitor various fields of interest. In most of these applications, such as structural health monitoring, pollution detection in water grids, and smart agriculture [1], we desire that the WSNs will function for a long time. However, the sensor nodes are typically powered by batteries, which severely limits the lifetime of WSNs.

Recently, energy harvesting paradigm has emerged as an attractive framework to prolong lifetime of WSNs [2], [3]. The environmental energy such as vibrations, solar radiation, and wind, can be harvested and stored at the rechargeable batteries of the sensor nodes to continuously supply energy to the nodes. Using this approach, it is possible to make a WSN immortal if the average energy consumption of every node is smaller or equal to its average harvested energy. However, the major drawback of relying on such ambient energy sources is that the power available is intermittent [4].

An attractive alternative is to use dedicated wireless charging where wireless energy transmitter are capable to transfer power to sensor nodes wirelessly. The result is that the charging of the nodes can be controlled by the system designer. In this paper, we consider such a system and investigate the optimal wireless energy transfer strategies and routing protocols when multiple energy transmitters equipped with

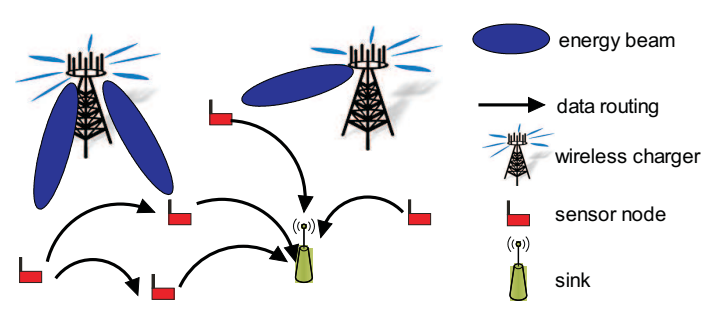

Fig. 1: A wireless sensor network with dedicated wireless energy chargers (base stations)

dedicated wireless energy transfer interfaces serve sensor nodes [5], as shown in Fig. 1. This system can be used in various applications such as pipeline monitoring, smart agriculture, and smart warehouse. To efficiently supply energy to the sensor nodes in longer distances, the transmitters use energy beamforming [6] to provide efficient energy beams. On the WSN side, the nodes make measurements and transmit the data to the sink via multi-hop. The monitoring performance is evaluated by the amount of measurements transmitted by the sensor nodes. To improve the monitoring performance of the whole WSN and to keep the WSN immortal, we jointly determine the beamforming vectors, the sampling rate and also the routing of the nodes, such that the sampled data can reach the sink in the most energy efficient way. To summarize, the contributions of this paper are as follows:

- We jointly consider the energy beamforming at the wireless chargers side and the data routing in the WSN side to maximize the monitoring performance and guarantee the immortality of the WSN, which, to the best of our knowledge, has not been studied before. This setup leads to a non-convex optimization formulation.

- We transform the original optimization problem above into to a semi-definite programming (SDP) problem, and show the strong duality of the SDP problem, which allows us to find the optimal solution for the SDP problem.

- We propose an algorithm to convert the optimal solution of the SDP problem to the solution of the original problem, and prove the optimality of solutions obtained with this procedure.

- We propose a low-complexity scheme where time-sharing between predetermined beamforming vectors is adopted.

- We numerically illustrate that the monitoring performance 
of a WSN with optimal energy beamforming is significantly better than the performance of the WSN without energy beamforming. These results also illustrate the close-to-optimal performance of the time-sharing predetermined beamforming scheme.

The rest of the paper is organized as follows. In Section II, we summarize the prior work on wireless energy beamforming and data routing. The joint energy beamforming and data routing problem is formulated in Section III. We propose the solution method for the optimization problem in Section IV, followed by the simulations in Section V. We conclude the paper and discuss the future works in Section VI.

Notation: For a vector $\boldsymbol{x}$ or a matrix $\boldsymbol{X},(\cdot)^{T}$ is the transpose of the vector or matrix, and $(\cdot)^{H}$ is the conjugate transpose of the vector or matrix. $\operatorname{tr}[\boldsymbol{X}]$ is the trace of square matrix $\boldsymbol{X}$. For a Hermitian matrix $\boldsymbol{X}$, the notation $\boldsymbol{X} \succeq 0$ means that $\boldsymbol{X}$ is positive semi-definite, and $\operatorname{Re}(\boldsymbol{X}), \operatorname{Im}(\boldsymbol{X})$ is the real and imaginary part of $\boldsymbol{X}$. Given a vector $\boldsymbol{x}$, the diagonalization $\operatorname{diag}[\boldsymbol{x}]$ constructs a matrix whose diagonal elements are $x_{1}, \ldots, x_{n}$.

\section{RELATED WORK}

Network utility maximization problems under energy harvesting from ambient energy sources have been investigated in a number of works [7], [8]. Reference [7] have considered the problem of scheduling the sensing time of nodes to optimize the total sensing utility of the WSN. Michelusi et. al. [8] have considered maximizing the long term data utility at the fusion center by scheduling the data transmission and discarding process based on the information of energy level and the energy harvesting process. Since the arrival of energy is intermittent and opportunistic in the case of energy harvesting from ambient sources [4], the performance of the WSN may be compromised in these systems. On the other hand, the WET framework allows us to have a better control on the charging process [4], [9]. In a WET system, a sensor node can store the energy received from electro-magnetic waves using rectifying antenna circuitry. Compared to energy harvesting from natural sources, such as solar radiation or vibrations, in WET framework it is possible to control the energy that will harvested by the sensor nodes by optimizing the transmission strategies of energy transmitters.

In this context, a throughput maximization problem over the energy allocation and WET time has been considered in [10]. The problem on joint design of energy beamforming vectors, energy allocation and the scheduling on WET durations to maximize the minimum throughput among different devices has been considered in [6]. For sensor networks, Liu et. al. [11] have considered maximizing the throughput of the wireless powered sensor network by controlling the energy transmission duration. They formulated a convex optimization and provided a closed form solution. In [12], the authors assumed that the base station forms a sharp energy beam to charge a sensor node in a timeslot, and studied the scheduling of the energy beams such that the WSN lifetime is maximized. Besides, Xie et. al. [13], [14] have considered a path planning problem for the charging vehicle to charge nodes, such that the WSN is immortal.

We note that the major energy consumption in WSN is due to data transmission. To maximize the network lifetime, one approach is to find a proper routing [15] for the sensor nodes. The energy consumption has been modelled as a function of the traffic flow routing in the seminal work [15], which provides us basic energy consumption model in this paper. Such model can well couple with the sampling rate of the sensor nodes, which is related to the monitoring performance of the WSN, as considered in [16].

Although optimal routing in WSN networks is a fundamental concern, only a limited number of works considered the routing problem together with the energy harvesting problem. When the sensor nodes can harvest energy from ambient environment, Sunny et. al. [17] have considered finding the routing and sensing for a WSN with energy harvesting capability to maximize the quality of monitoring. They formulated the problem as a resource allocation problem and provided a near optimal solution algorithm. Different from their work, we consider the case where dedicated energy transmitters are charging the WSN. Thus, besides the routing of the WSN, we also need to optimize over the wireless energy transmission part, more specifically, we need to design the energy beamforming vectors and their time durations. Different from some works on WET where energy is broadcasted [11], [18] with a fixed power, the energy transmitter we consider form sharp beams to improve the received energy at the sensor nodes, which however makes the optimization problem more challenging. To the best of our knowledge, this is the first paper that jointly considers the energy beamforming and the data routing problem.

\section{System Model ANd Problem Formulation}

We consider a routing and energy beamforming problem for a wirelessly powered wireless sensor network. Specifically, a wireless sensor network (WSN) that consists of multiple sensor nodes is deployed to monitor a field of interest, and multiple wireless chargers, which can be base stations, form energy beams to charge to the nodes, as shown in Fig. 1. Each node $v_{i}$ makes the measurement with sampling rate $w_{i}$. Then, it transmits the data to the sink in a multi-hop way. To allow the entire WSN to be immortal, each node is powered by a rechargeable battery, where the energy comes from the energy beams from multiple wireless chargers. Such a network structure can be applied to multiple applications, such as smart agriculture, smart pipeline monitoring, smart warehouse.

We assume that there are $N$ sensor nodes including the sink in the WSN. We denote $q_{i j}$ the data flow from $v_{i}$ to $v_{j}$, and $e_{i j}^{O}$ the energy cost of sending a data unit. Then, the energy consumption of a node $v_{i}$ is given by

$$
E_{i}^{U}=\sum_{k \in S_{i}^{\text {out }}} e_{i k}^{O} q_{i k},
$$


where $\mathcal{S}_{i}^{\text {out }}$ is the set of succeeding neighbor nodes of $v_{i}{ }^{1}$. We define $\mathcal{S}_{i}^{\text {in }}$ as the set of preceding neighbor nodes of $v_{i}$.

We let $n_{\mathrm{ET}}$ be the number of wireless chargers. Each charger has $M \gg N$ antennas, such that energy beams can be formed at each charger to improve the energy harvested at the sensor nodes. Note that the energy beam of a charger can be time-varying, i.e., a charger $l$ can form energy beam vectors $\boldsymbol{u}_{l, 1}, \boldsymbol{u}_{l, 2}, \ldots, \boldsymbol{u}_{l, j}, \ldots$, and for any beam vector $\boldsymbol{u}_{l, j} \in \mathbb{C}^{M}$. We assume here that the number of the beam vectors is larger or equal to $M$. We denote $t_{l, j}$ the average time the charger transmits beam $\boldsymbol{u}_{l, j}$. Let the channel from charger $l$ to node $i$ be $\boldsymbol{g}_{l, i} \in \mathbb{C}^{M \times 1}$. Then, similar to [6], the average energy received by node $i$ is expressed as

$$
E_{i}^{R}=\sum_{l=1}^{n_{E T}} \sum_{j} \eta t_{l, j} \mathbb{E}_{g}\left[\boldsymbol{g}_{l, i}^{H} \boldsymbol{u}_{l, j} \boldsymbol{u}_{l, j}^{H} \boldsymbol{g}_{l, i}\right],
$$

where the expectation is over the channels $\boldsymbol{g}_{l, i}, \eta$ is the energy conversion efficiency, and the receiver noise is ignored, since it is negligible for energy receivers [6].

We assume that the battery buffers of the sensor nodes are large enough, such that the WSN is immortal if $E_{i}^{R} \geq E_{i}^{U}, \forall i$. Thus, the sampling rate and the routing of the nodes are constrained by the total energy it receives. For the WSN, we want to have the sampled data as much as possible, but not to have some sensors sampling with very low rate (balancing issue). Thus, we denote $F(\boldsymbol{w})=\sum_{i=1}^{N} \log \left(1+w_{i}\right)$ the monitoring performance of the $\mathrm{WSN}^{2}$, which is assumed to be a concave function of $\boldsymbol{w}=\left[w_{1}, w_{2}, \ldots, w_{N}\right]^{T}$. We stack $q_{i j}$ s to become a column vector $\boldsymbol{q} \in \mathbb{R}^{L}$, where $L$ is the number of candidate data routing links of the whole WSN. Then, the considered problem can be formulated as:

$$
\begin{array}{cl}
\max _{\boldsymbol{w}, \boldsymbol{q}, \boldsymbol{u}, \boldsymbol{t}} & F(w) \\
\text { s.t. } & w_{i}+\sum_{j \in S_{i}^{\text {in }}} q_{j i}-\sum_{k \in S_{i}^{\text {out }}} q_{i k}=0, \quad \forall i \\
& E_{i}^{U} \leq E_{i}^{R}, \quad \forall i \\
& \sum_{j} t_{l, j} \boldsymbol{u}_{l, j}^{H} \boldsymbol{u}_{l, j} \leq P_{l}, \forall l \\
& \sum_{l=1}^{n_{E T}} \sum_{j} t_{l, j} \boldsymbol{u}_{l, j}^{H} \boldsymbol{u}_{l, j} \leq P, \\
& \sum_{j} t_{l, j}=1, \quad \forall l, \\
& \boldsymbol{w}, \boldsymbol{q}, \boldsymbol{t} \geq 0,
\end{array}
$$

where $\boldsymbol{w}, \boldsymbol{q}, \boldsymbol{t}$ are all non-negative, Constraint (3b) represents the conservation of the data flow, Constraint (3c) ensures the immortality of the WSN, Constraint (3d) provides the power constraint for each charger, Constraint (3e) is the

\footnotetext{
${ }^{1}$ Notice that here we omit the energy cost on sensing and receiving data since they are much smaller than the energy cost on transmitting data. However, it can be shown that, when the sensing and receiving energy is also considered, the proposed algorithm still applies.

${ }^{2}$ Alternatively, we can let the objective function be $F(\boldsymbol{w})=\sum_{i} \alpha_{i} w_{i}$ which means the weighted number of sampling rate of the nodes.
}

TABLE I: Major notations used in the paper

\begin{tabular}{|c|l|}
\hline symbols & meanings \\
\hline $\mathcal{S}^{\text {in }}$ and $\mathcal{S}^{\text {out }}$ & set of preceding and succeeding neighbor nodes of $v_{i}$ \\
\hline $\boldsymbol{B}$ & candidate routing tables of the sensor nodes \\
\hline$E_{i}^{U}$ and $E_{i}^{R}$ & energy consumption matrix of the nodes \\
\hline$F(\boldsymbol{w})$ & monitoring performance function on $\boldsymbol{w}$ \\
\hline $\boldsymbol{I}_{i}$ & $\begin{array}{l}\text { a matrix with 1 only at the } i \text {-th element of } \\
\text { its diagonal and with } 0 \text { for the others }\end{array}$ \\
\hline $\boldsymbol{K}_{l, i}$ & $\mathbb{E}_{g}\left[\boldsymbol{g}_{l, i} \boldsymbol{g}_{l, i}^{H}\right]$ \\
\hline$L$ & number of candidate links \\
\hline$M$ & number of antennas of each wireless charger \\
\hline$N$ & number of sensor nodes \\
\hline$P$ and $P_{l}$ & $\begin{array}{l}\text { the constraint of the total transmitted power of } \\
\text { all the chargers and charger } l\end{array}$ \\
\hline $\boldsymbol{U}_{l}$ & $\sum_{j} t_{l, j} \boldsymbol{u}_{l, j} \boldsymbol{u}_{l, j}^{H}$ \\
\hline $\boldsymbol{W}_{e_{i j}}$ & diag $\boldsymbol{w}+\mathbf{1})$ \\
\hline $\boldsymbol{g}_{l, i}$ & energy cost of sending one data unit from $v_{i}$ to $v_{j}$ \\
\hline$n_{E T}$ & channel from charger $l$ to node $i$ \\
\hline$q_{i j}$ & number of wireless chargers \\
\hline$t_{l, j}$ & data flow from $v_{i}$ to $v_{j}$ \\
\hline $\boldsymbol{u}_{l, j}$ & average time the charger $l$ transmit beam $\boldsymbol{u}_{l, j}$ \\
\hline$v_{i}$ & the $j$-th energy beam vector of charger $l$ \\
\hline$w_{i}$ & sampling rate of $v_{i}$ \\
\hline$\eta$ & energy conversion efficiency \\
\hline
\end{tabular}

total power constraint from the safety consideration, and Constraint (3f) means that, for each charger $l$, the summation of the percentage of time to form energy beam $\boldsymbol{u}_{l, j}$ is 1 . We note that the problem is non-convex due to the non-convex quadratic constraints $(3 \mathrm{c})$ even if $t_{l, j}$ were fixed. However, we propose an algorithm to find the optimal solution for the problem, as shown in the next section.

For a better readability, we provide the major notations of the paper in Table I.

\section{SOlution ApproACH}

In this section, we will propose a solution method for Problem (3), and then analyze a special case of the problem.

\section{A. Algorithm based on SDP}

The idea of the solution algorithm is to first transform the original problem to a convex optimization problem, whose optimal solution can be found efficiently. Then, we will show that the optimal solution of the convex problem is also the optimal solution for the original problem.

For simplicity, we construct a matrix $\boldsymbol{A}=\left\{a_{i j}\right\} \in \mathbb{R}^{N \times L}$ that corresponds to the candidate routing table of the nodes, where $a_{i, j}=1$ if there is a link from $v_{j}$ to $v_{i} ; a_{i, j}=-1$ if there is a link from $v_{i}$ to $v_{j}$; otherwise, $a_{i, j}=0$. Then, we can re-write Constraint (3b) as $\boldsymbol{w}+\boldsymbol{A} \boldsymbol{q}=\mathbf{0}$, where $\boldsymbol{q} \in \mathbb{R}^{L \times 1}$ is the vector of flow. Similarly, we use $\boldsymbol{B}_{i}=\left\{b_{i, j}\right\} \in \mathbb{R}^{1 \times L}$ to represent the energy consumption for each communication pair that starts with node $v_{i}$, i.e., $b_{i, j}=e_{i, j}^{O}$ if there is a candidate link from $v_{i}$ to $v_{j}$. Then, $E_{i}^{U}$ can be written as $\boldsymbol{B}_{\boldsymbol{i}} \boldsymbol{q}$. By stacking up the row vectors $\boldsymbol{B}_{i}$, we construct the energy 
consumption matrix of the nodes, denoted by $\boldsymbol{B} \in \mathbb{R}^{N \times L}$. Then, we can re-write Constraint (3c) as follows:

$$
\begin{aligned}
\boldsymbol{B}_{i} \boldsymbol{q} & \leq \sum_{l=1}^{n_{E T}} \sum_{j} \eta t_{l, j} \mathbb{E}_{g}\left[\boldsymbol{g}_{l, i}^{H} \boldsymbol{u}_{l, j} \boldsymbol{u}_{l, j}^{H} \boldsymbol{g}_{l, i}\right] \\
& =\sum_{l=1}^{n_{\mathrm{ET}}} \sum_{j} \eta t_{l, j} \operatorname{tr}\left[\boldsymbol{K}_{l, i} \boldsymbol{u}_{l, j} \boldsymbol{u}_{l, j}^{H}\right] \\
& =\eta \sum_{l=1}^{n_{\mathrm{ET}}} \operatorname{tr}\left[\boldsymbol{K}_{l, i} \sum_{j} t_{l, j} \boldsymbol{u}_{l, j} \boldsymbol{u}_{l, j}^{H}\right]
\end{aligned}
$$

where $\boldsymbol{K}_{l, i}=\mathbb{E}_{g}\left[\boldsymbol{g}_{l, i} \boldsymbol{g}_{l, i}^{H}\right]$ and it is a Hermitian positive semidefinite matrix. Similarly, Constraint (3d) and Constraint (3e) can be re-written similarly as $\operatorname{tr}\left[\sum_{j} t_{l, j} \boldsymbol{u}_{l, j} \boldsymbol{u}_{l, j}^{H}\right] \leq P, \forall l$ and $\sum_{l=1}^{n_{E T}} \operatorname{tr}\left[\sum_{j} t_{l, j} \boldsymbol{u}_{l, j} \boldsymbol{u}_{l, j}^{H}\right] \leq P$.

Now, if we substitute $\sum_{j} t_{l, j} \boldsymbol{u}_{l, j} \boldsymbol{u}_{l, j}^{H}$ by a Hermitian positive semi-definite matrix $\boldsymbol{U}_{l}$, the original Problem (3) can be re-written as follows:

$$
\begin{aligned}
\underset{\boldsymbol{w}, \boldsymbol{q}, \boldsymbol{u}, \boldsymbol{t}}{\max } & F(\boldsymbol{w}) \\
\text { s.t. } & \boldsymbol{w}+\boldsymbol{A} \boldsymbol{q}=\mathbf{0}, \\
& \boldsymbol{B}_{i} \boldsymbol{q} \leq \eta \sum_{l=1}^{n_{E T}} \operatorname{tr}\left[\boldsymbol{K}_{l, i} \boldsymbol{U}_{l}\right], \quad \forall i \\
& \operatorname{tr}\left[\boldsymbol{U}_{l}\right] \leq P_{l}, \quad \forall l \\
& \sum_{E T}^{n_{E}} \operatorname{tr}\left[\boldsymbol{U}_{l}\right] \leq P, \\
& \boldsymbol{U}_{l=1} \succeq 0, \forall l, \\
& \boldsymbol{w} \geq 0, \boldsymbol{q} \geq 0, \boldsymbol{t} \geq 0, \\
& \boldsymbol{U}_{l}=\sum_{j} t_{j} \boldsymbol{u}_{l, j} \boldsymbol{u}_{l, j}^{H}, \quad \forall l, \\
& \sum_{j} t_{j}=1 .
\end{aligned}
$$

Notice that if we relax Constraints (4h) - (4i), Problem (4) turns to be the following one:

$$
\begin{aligned}
\min _{\boldsymbol{w}, \boldsymbol{q}, \boldsymbol{U}_{l}} & -\sum_{i} \log \left(1+w_{i}\right) \\
\text { s.t. } & (4 \mathrm{~b}),(4 \mathrm{c}),(4 \mathrm{~d}),(4 \mathrm{e}),(4 \mathrm{f}),(4 \mathrm{~g}),
\end{aligned}
$$

which is convex as described by the following proposition:

Proposition 1: Problem (5) is equivalent to a convex semidefinite programming problem with a log determinant term in objective function.

Proof: Let $\boldsymbol{W}=\operatorname{diag}(\boldsymbol{w}+\mathbf{1}), \boldsymbol{Q}=\operatorname{diag}(\boldsymbol{q})$. Since $\boldsymbol{w} \geq 0$, we have that $\boldsymbol{W} \succ 0$. Then, the objective function of Problem (5) can be written as $F(\boldsymbol{W})=\log \operatorname{det}(\boldsymbol{W})$.

Constraint (4b) is equivalent to $\operatorname{tr}\left[\boldsymbol{I}_{i} \boldsymbol{W}\right]+\operatorname{tr}\left[\operatorname{diag}\left(\boldsymbol{A}_{i}\right) \boldsymbol{Q}\right]=$ 1 , where $\boldsymbol{A}_{i}$ is the $i$-th row of $\boldsymbol{A}, \boldsymbol{I}_{i}$ is a matrix with 1 only at the $i$-th element of its diagnose, and with 0 for the other elements. Constraints (4c) are equivalent to $\operatorname{tr}\left[\operatorname{diag}\left(\boldsymbol{B}_{i}\right) \boldsymbol{Q}\right]-$ $\sum_{l=1}^{n_{E T}} \operatorname{tr}\left[\boldsymbol{K}_{l, i} \boldsymbol{U}_{l}\right] \leq 0^{3}$.

To summarize, Problem (5) is equivalent to the following form:

$$
\begin{array}{cl}
\min _{\boldsymbol{W}, \boldsymbol{Q}, \boldsymbol{U}_{l}} & -F(\boldsymbol{W})=-\log \operatorname{det}(\boldsymbol{W}) \\
\text { s.t. } & \operatorname{tr}\left[\boldsymbol{I}_{i} \boldsymbol{W}\right]+\operatorname{tr}\left[\operatorname{diag}\left(\boldsymbol{A}_{i}\right) \boldsymbol{Q}\right]=1, \forall i \\
& \operatorname{tr}\left[\operatorname{diag}\left(\boldsymbol{B}_{i}\right) \boldsymbol{Q}\right]-\eta \sum_{l} \operatorname{tr}\left[\boldsymbol{K}_{l, i} \boldsymbol{U}_{l}\right] \leq 0, \forall i \\
& \operatorname{tr}\left[\boldsymbol{U}_{l}\right] \leq P_{l}, \forall l \\
& \sum_{l=1}^{n_{E T}} \operatorname{tr}\left[\boldsymbol{U}_{l}\right] \leq P, \\
& \boldsymbol{W} \succ \mathbf{0}, \boldsymbol{Q} \succeq \mathbf{0}, \boldsymbol{U}_{l} \succeq \mathbf{0}
\end{array}
$$

Since the objective function is convex (on $\mathcal{S}_{++}^{N}$ ), and the feasible region is convex, we have that Problem (5) is convex, and we can consider it as a SDP problem whose objective function has a log determinant term.

Since Problem (6) is a SDP problem, we can solve it by efficient numerical methods, such as interior-point point methods. Thus, the idea here is to first find the optimal solution for Problem (5), denoted by $\boldsymbol{w}_{\text {relax }}^{*}, \boldsymbol{q}_{\text {relax }}^{*}, \boldsymbol{U}_{\text {relax }}^{*}$. Then, based on $\boldsymbol{U}_{\text {relax }}^{*}$, we find $\boldsymbol{u}, \boldsymbol{t}$ that satisfy Constraints (4h) - (4i) in the second step. If there exists $\boldsymbol{u}_{\text {relax }}^{*}, \boldsymbol{t}^{*}$ that satisfies the constraints, then $\boldsymbol{w}_{\text {relax }}^{*}, \boldsymbol{q}_{\text {relax }}^{*}, \boldsymbol{u}_{\text {relax }}^{*}, \boldsymbol{t}^{*}$ is also the optimal solution for Problem (4).

Next, we need to show the strong duality of the problem, such that the duality gap is 0 and we can achieve the optimal solution for Problem (6) with any sufficiently small error $\epsilon>0$ in time $O\left(\left(L+N+n_{E T} M\right)^{4.5} \log (1 / \epsilon)\right)$ [19]. Note that the strong duality in linear programming and in convex SDP problems are different, where for the latter both primal problem and the dual problem must have a non-empty interior [20]. We first write the dual problem of Problem (6) as follows:

$$
\begin{aligned}
\max _{\boldsymbol{Z} \succ 0, \boldsymbol{y}} & \boldsymbol{b}^{T} \boldsymbol{y}+\log \operatorname{det}(\boldsymbol{Z}) \\
\text { s.t. } & \sum_{i} y_{1 i} \boldsymbol{I}_{i}+\boldsymbol{Z}=\mathbf{0}, \\
& \sum_{i} y_{1 i} a_{i j}+\sum_{i} y_{2 i} b_{i j} \leq 0, \forall j \\
& \eta \sum_{i=1}^{N} y_{2 i} \boldsymbol{K}_{l, i}-\left(y_{3 l}+y_{4}\right) \boldsymbol{I} \succeq 0, \forall l \\
& y_{2 i} \leq 0, y_{3 l} \leq 0, y_{4} \leq 0, \forall i, l
\end{aligned}
$$

where $y_{1 i}$ corresponds to Constraint (6b), $y_{2 i}$ corresponds to Constraint (6c), $y_{3 l}$ corresponds to Constraint (6d), $y_{4}$ corresponds to Constraint (6e).

\footnotetext{
${ }^{3}$ Here, for any complex positive semi-definite matrix $\boldsymbol{X}$, we can convert it to a real positive semi-definite matrix by

$$
\boldsymbol{X}^{\prime}=\left[\begin{array}{cc}
\operatorname{Re}(\boldsymbol{X}) & -\operatorname{Im}(\boldsymbol{X}) \\
\operatorname{Im}(\boldsymbol{X}) & \operatorname{Re}(\boldsymbol{X})
\end{array}\right]
$$
}


Then, we have the following proposition:

Proposition 2: Consider Problem (6) and its dual (7), where $\boldsymbol{A}, \boldsymbol{B}$ are constructed according to the topology of a connected $\mathrm{WSN}^{4}, P>0, P_{l}>0$. Strong duality holds, i.e., for both of the problems there exist strictly feasible solutions. ${ }^{5}$

Proof: The proof consists of checking the existence of the strictly feasible solutions for each problem. Since the WSN is connected, the elements in $\boldsymbol{B}_{i}$ are bounded and positive.

For Problem (6), we can set $\boldsymbol{U}_{l}=\left(\min \left\{1, P / \sum P_{l}\right\}-\right.$ $\varepsilon) P_{l} \boldsymbol{I}$. As $\boldsymbol{K}_{l, i}$ is positive semi-definite, and $\boldsymbol{K}_{l, i} \neq \mathbf{0}$, we have $\operatorname{tr}\left[\boldsymbol{K}_{l, i}\right]>0$. Thus, it is straightforward that we can find a small enough routing decision $Q$, such that $0<\operatorname{tr}\left[\operatorname{diag}\left(\boldsymbol{B}_{i}\right) \boldsymbol{Q}\right]<\eta \sum_{l}\left(\min \left\{1, P / \sum P_{l}\right\}-\varepsilon\right) \operatorname{tr}\left[\boldsymbol{K}_{l, i}\right]$. It means that there exists strictly feasible solutions for Problem (6).

For Problem (7), Constraints (7b) requires that $y_{1 i}<0, \forall i$. We can set $y_{1 i}=-\varepsilon_{1}<0, \forall i$, where $\varepsilon_{1}>0$. Since $\boldsymbol{A}$ corresponds to the candidate routing table, each column of which has at most one 1 and one -1 , Then, we have that $\sum_{i} y_{1 i} a_{i j} \leq \varepsilon_{1}$. Since $\boldsymbol{B}$ corresponds to the energy consumption for each candidate link, we have that $b_{i j} \geq 0$, and $\sum_{i} b_{i j}>0$. Thus, we can set $y_{2 i}=-\varepsilon_{1} / \min _{j} \sum_{k} b_{k j}-\varepsilon_{2}, \forall i$, where $\varepsilon_{2}>0$, such that $\sum_{i} y_{2 i} b_{i j} \leq-\varepsilon_{1}$ and Constraint (7c) strictly holds. For Constraint (7d), it is also possible to find small enough $y_{3 l}, y_{4}$, such that $y_{3 l}+y_{4}$ is smaller than the smallest eigenvalue of $\eta \sum_{i=1}^{N} y_{2 i} \boldsymbol{K}_{l, i}, \forall l$, which makes Constraints (7d) strictly hold. Thus, there exists strictly feasible solution for Problem (7).

Thus, we have that for Problem (6) and its dual Problem (7), there exists strictly feasible solution, which completes the proof.

From this proposition, we can achieve a solution with a sufficient small error $\epsilon>0$ to the optimal solution of Problem (6) in time $\log (1 / \epsilon)$ [19]. We consider this approximate solution to be global optimal since the error can be sufficient small. Next, we are going to find the optimal solution of Problem (4) based on the optimal solution of Problem (6), which are denoted by $\left(\boldsymbol{w}_{\text {relax }}^{*}, \boldsymbol{q}_{\text {relax }}^{*}, \boldsymbol{U}_{\text {relax }}^{*}\right)$.

Recalling that $\boldsymbol{U}_{l}, \forall l$ is positive semi-definite, we have that all the eigenvalues of $\boldsymbol{U}_{l, \text { relax }}^{*}$ are non-negative. Thus, we can let $t_{l, j}=\lambda_{l, j} / \sum_{i} \lambda_{l, i}$ and $\boldsymbol{u}_{l, j}=\sqrt{\sum_{i} \lambda_{l, i}} \boldsymbol{d}_{l, j}$ to be the solution, where $\lambda_{l, j}, \boldsymbol{d}_{l, j}$ are the eigen value and corresponding eigen vector of $\boldsymbol{U}_{l \text {,relax. Therefore, we }}^{*}$ have that the time-splitting beamforming problem can be solved by turning the problem into a convex optimization problem, then making the spectrum decomposition, and rescaling the results. The algorithm to find the optimal solution for Problem (3) is summarized in Algorithm 1. We have the following proposition of Algorithm 1:

Proposition 3: Consider a feasible optimization Problem (4), where $\boldsymbol{K}_{l, i}$ is positive semi-definite. We

\footnotetext{
${ }^{4}$ It means that, for each column of $\boldsymbol{A}$, there exists at most one 1 and one -1 , whereas the other elements are 0 . For $\boldsymbol{B}$, all its elements are non-negative.

${ }^{5}$ It is also sufficient to show that the proposition holds for the case where $F(\boldsymbol{w})=\sum \alpha_{i} w_{i}$
}

have that Algorithm 1 achieves a global optimal solution for Problem (4).

Proof: Denote $\boldsymbol{w}_{\text {relax }}, \boldsymbol{q}_{\text {relax }}, \boldsymbol{u}_{\text {relax }}, \boldsymbol{t}_{\text {relax }}$ the output of Algorithm (1). First we prove that $\boldsymbol{w}_{\text {relax }}, \boldsymbol{q}_{\text {relax }}, \boldsymbol{u}_{\text {relax }}, \boldsymbol{t}_{\text {relax }}$ is feasible for Problem (4) as follows.

According to Proposition 1, Problem (5) is a convex problem. Thus, the optimal solution for Problem (5), $\left(\boldsymbol{w}_{\text {relax }}^{*}, \boldsymbol{q}_{\text {relax }}^{*} \boldsymbol{U}_{\text {relax }}^{*}\right)$, is achievable. Thus, we have that $\boldsymbol{B}_{i} \boldsymbol{q}_{\text {relax }}^{*} \leq \eta \sum_{l} \operatorname{tr}\left[\boldsymbol{K}_{l, i} \boldsymbol{U}_{l, \text { relax }}^{*}\right], \operatorname{tr}\left[\boldsymbol{U}_{l, \text { relax }}^{*}\right] \leq P_{l}$, and $\sum_{l} \operatorname{tr}\left[\boldsymbol{U}_{l, \text { relax }}^{*}\right] \leq P$. As $\boldsymbol{u}_{l, j \text {,relax }}=\sqrt{\sum_{i} \lambda_{l, i}} \boldsymbol{d}_{l, j}, t_{l, j, \text { relax }}=$ $\lambda_{l, j} / \sum_{i} \lambda_{l, i}$, where $\lambda_{l, i}$ and $\boldsymbol{d}_{l, i}$ are the eigenvalue and corresponding eigen vector of $\boldsymbol{U}_{l, \text { relax }}^{*}$, we have that

$$
\begin{aligned}
P_{l} \geq \operatorname{tr}\left[\boldsymbol{U}_{l, \text { relax }}^{*}\right] & =\sum_{i} \lambda_{l, i} \boldsymbol{d}_{l, i}^{H} \boldsymbol{d}_{l, i} \\
& =\sum_{i} t_{l, i, \text { relax }} \boldsymbol{u}_{l, i, \text { relax }}^{H} \boldsymbol{u}_{l, i, \text { relax }},
\end{aligned}
$$

where the first equality holds due to that $\boldsymbol{U}_{\text {relax }}^{*}$ is positive semi-definite, which is diagonalizable. Therefore, $t_{l, i, \text { relax }}, \boldsymbol{u}_{l, i, \text { relax }}$ satisfies Constraints (4d), (4h) and (4i). Similarly, we have that

$$
P \geq \sum_{l=1}^{n_{E T}} \operatorname{tr}\left[\boldsymbol{U}_{l, \text { relax }}^{*}\right]=\sum_{l=1}^{n_{E T}} \sum_{i} t_{l, i, \text { relax }} \boldsymbol{u}_{l, i, \text { relax }}^{H} \boldsymbol{u}_{l, i, \text { relax }}
$$

and

$$
\begin{aligned}
\boldsymbol{B}_{i} \boldsymbol{q}_{\mathrm{relax}}^{*} & \leq \eta \operatorname{tr}\left[\boldsymbol{K}_{l, i} \boldsymbol{U}_{l, \text { relax }}^{*}\right] \\
& =\eta \operatorname{tr}\left[\boldsymbol{K}_{l, i} \sum_{i} t_{l, i, \text { relax }} \boldsymbol{u}_{l, j, \text { relax }} \boldsymbol{u}_{l, i, \text { relax }}^{H}\right],
\end{aligned}
$$

which means that $\left(\boldsymbol{w}_{\text {relax }}^{*}, \boldsymbol{q}_{\text {relax }}^{*} \boldsymbol{t}_{\text {relax }}, \boldsymbol{u}_{\text {relax }}\right)$ satisfies Constraints $(4 \mathrm{c})$ and $(4 \mathrm{e})$.

Furthermore, since $\boldsymbol{U}_{l, \text { relax }}^{*}$ is positive semi-definite, its eigenvalue $\lambda_{l, i}$ is nonnegative and real for all $i$, which means that $t_{l, i}$ is nonnegative and real for all $i$. Thus, $\boldsymbol{t}_{\text {relax }}$ satisfies Constraint (4i) and $\boldsymbol{t} \geq 0$. Therefore, $\left(\boldsymbol{w}_{\text {relax }}, \boldsymbol{q}_{\text {relax }}, \boldsymbol{t}_{\text {relax }}, \boldsymbol{u}_{\text {relax }}\right)$ is a feasible solution of Problem (4).

For the optimality, it is easy to show that, suppose there exists a feasible solution $\left(\boldsymbol{w}_{o}, \boldsymbol{q}_{o}, \boldsymbol{t}_{o}, \boldsymbol{u}_{o}\right)$, such that $F\left(\boldsymbol{w}_{0}\right)>F\left(\boldsymbol{w}_{\text {relax }}\right)$, then we can construct $\boldsymbol{U}_{l, o}=$ $\sum_{i} \boldsymbol{t}_{l, i} \boldsymbol{u}_{l, i o} \boldsymbol{u}_{l, i o}^{H}$, such that $\boldsymbol{x}_{o}, \boldsymbol{U}_{l, o}$ is feasible for Problem (5). Then, $F\left(\boldsymbol{w}_{0}\right)>F\left(\boldsymbol{w}_{\text {relax }}\right)=F\left(\boldsymbol{w}_{\text {relax }}^{*}\right)$ is contradicted to that $\left(\boldsymbol{w}_{\text {relax }}^{*}, \boldsymbol{q}_{\text {relax }}^{*}, \boldsymbol{U}_{\text {relax }}\right)$ is the optimal solution for Problem (5). Thus, $\left(\boldsymbol{w}_{\text {relax }}, \boldsymbol{q}_{\text {relax }}, \boldsymbol{t}_{\text {relax }}, \boldsymbol{u}_{\text {relax }}\right)$ is an optimal solution for Problem (4), and completes the proof.

Recall that Problem (4) is equivalent to Problem (3). Algorithm 1 achieves a global optimal solution for Problem (3).

\section{B. Special Case: Predetermined beamforming vectors}

In this subsection, we are going to discuss a special case of Problem (3) where the beamforming vectors of the chargers are pre-determined, whereas the power and the time duration for each beam is to be optimized. In this case, we suppose 
that, for charger $l$, it has $M$ pre-determined beams, which are denoted by $\boldsymbol{u}_{l, j}, 1 \leq j \leq M$, and $\left\|\boldsymbol{u}_{l, j}\right\|^{2}=1$. We denote the power and average time of beam $\boldsymbol{u}_{l, j}$ by $p_{l, j}$ and $t_{l, j}$. Then, the optimization problem is

$$
\begin{array}{cl}
\max _{\boldsymbol{w}, \boldsymbol{q}, \boldsymbol{p}, \boldsymbol{t}} & F(\boldsymbol{w}) \\
\text { s.t. } & \boldsymbol{w}+\boldsymbol{A} \boldsymbol{q}=\mathbf{0} \\
& \boldsymbol{B}_{i} \boldsymbol{q} \leq \sum_{l=1}^{n_{\mathrm{ET}}} \sum_{j=1}^{M} \eta p_{l, j} t_{l, j} \operatorname{tr}\left[\boldsymbol{K}_{l, i} \boldsymbol{u}_{l, j} \boldsymbol{u}_{l, j}^{H}\right], \forall i \\
& \sum_{l=1}^{n_{\mathrm{ET}}} \sum_{j=1}^{M} p_{l, j} t_{l, j} \leq P \\
& \sum_{j=1}^{M} t_{l, j}=1, \forall l \\
& \sum_{j=1}^{M} p_{l, j} t_{l, j} \leq P_{l}, \forall l \\
& \boldsymbol{w} \geq 0, \boldsymbol{q} \geq 0, \boldsymbol{p} \geq 0, \boldsymbol{t} \geq 0 .
\end{array}
$$

Problem (9) is non-convex, due to the multiplication of the variables in the constraints. However, if we introduce a new variable $y_{l, j}$ to represent $p_{l, j} t_{l, j}$. Then, we can find the optimal solution of Problem (9). The approach consists of two steps. First, we temporally relax Constraints (9e), and solve the following problem:

$$
\begin{array}{rl}
\max _{\boldsymbol{w}, \boldsymbol{q}, \boldsymbol{y}} & F(\boldsymbol{w}) \\
\text { s.t. } & \boldsymbol{w}+\boldsymbol{A} \boldsymbol{q}=\mathbf{0} \\
& \boldsymbol{B}_{i} \boldsymbol{q} \leq \sum_{l=1}^{n_{\mathrm{ET}}} \sum_{j=1}^{M} \eta y_{l, j} \operatorname{tr}\left[\boldsymbol{K}_{l, i} \boldsymbol{u}_{l, j} \boldsymbol{u}_{l, j}^{H}\right], \forall i \\
& \sum_{l=1}^{n_{\mathrm{ET}}} \sum_{j=1}^{M} y_{l, j} \leq P \\
& \sum_{j=1}^{M} y_{l, j} \leq P_{l}, \forall l \\
& \boldsymbol{w} \geq 0, \boldsymbol{q} \geq 0, \boldsymbol{y} \geq 0,
\end{array}
$$

which is convex. Suppose the optimal solution for Problem (10a) is $\left(\boldsymbol{w}^{*}, \boldsymbol{q}^{*}, \boldsymbol{y}^{*}\right)$. Then, in the second step, we need to find the feasible $t_{l, j}$ and $p_{l, j}, \forall l, j$, such that the following equations are satisfied:

$$
\left\{\begin{array}{l}
p_{l, j} t_{l, j}=y_{l, j}^{*}, \quad \forall l, j \\
\sum_{i=1}^{M} t_{l, j}=1, \quad \forall l \\
0 \leq p_{l, j}, 0 \leq t_{l, j}, \quad \forall l, j .
\end{array}\right.
$$

One possible solution that satisfies Equations (11) is given by $p_{l, j}=M y_{l, j}^{*}, t_{l, j}=1 / M, \forall l, j$. Then, we have the following proposition:

Proposition 4: Consider feasible optimization Problem (9). If $\left(\boldsymbol{w}^{*}, \boldsymbol{q}^{*}, \boldsymbol{y}^{*}\right)$ is the optimal solutions for Problem (10a),

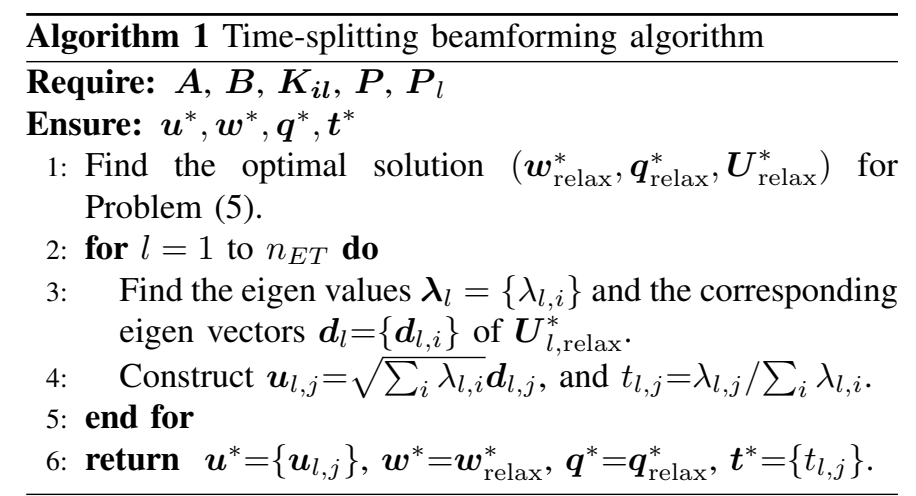

then $\left(\boldsymbol{w}^{*}, \boldsymbol{q}^{*}, \boldsymbol{p}=M \boldsymbol{y}^{*}, \boldsymbol{t}=(1 / M) \mathbf{1}^{T}\right)$ is one of the optimal solution for Problem (9).

Proof: The proof consists in checking the feasibility and the optimality.

Regarding the feasibility, Constraints (9b) - (9d), and (9f) can be easily achieves due to that $\left(\boldsymbol{w}^{*}, \boldsymbol{q}^{*}, \boldsymbol{y}^{*}\right)$ is the feasible solution for Problem (10a), and that $p_{l, j} t_{l, j}=y_{l, j}^{*}$. Constraints (9e) holds due to $t_{l, j}=1 / M, \forall i, l$. Thus, $\left(\boldsymbol{w}^{*}, \boldsymbol{q}^{*}, \boldsymbol{p}=M \boldsymbol{y}^{*}, \boldsymbol{t}=(1 / M) \mathbf{1}^{T}\right)$ is a feasible solution of Problem (9).

Regarding the optimality, suppose there is another feasible solution $\left(\boldsymbol{w}^{\prime}, \boldsymbol{q}^{\prime}, \boldsymbol{p}^{\prime}, \boldsymbol{t}^{\prime}\right)$ such that $F\left(\boldsymbol{w}^{\prime}\right)>F\left(\boldsymbol{w}^{*}\right)$. Then, we can construct $\boldsymbol{y}^{\prime}=\left\{y_{l, j}^{\prime}\right\}$ where $y_{l, j}^{\prime}=p_{l, j}^{\prime} t_{l, j}^{\prime}$, such that $\left(\boldsymbol{w}^{\prime}, \boldsymbol{q}^{\prime}, \boldsymbol{y}^{\prime}\right)$ is feasible for Problem (10a). Then, $F\left(\boldsymbol{w}^{\prime}\right)>F\left(\boldsymbol{w}^{*}\right)$ is contradicted to that $\left(\boldsymbol{w}^{*}, \boldsymbol{q}^{*}, \boldsymbol{y}^{*}\right)$ is the optimal solution for Problem (10a). Therefore, there exists no $\left(\boldsymbol{w}^{\prime}, \boldsymbol{q}^{\prime}, \boldsymbol{p}^{\prime}, \boldsymbol{t}^{\prime}\right)$ such that $F\left(\boldsymbol{w}^{\prime}\right)>F\left(\boldsymbol{w}^{*}\right)$.

To sum up, $\left(\boldsymbol{w}^{*}, \boldsymbol{q}^{*}, \boldsymbol{p}=M \boldsymbol{y}^{*}, \boldsymbol{t}=(1 / M) \mathbf{1}^{T}\right)$ is one of the global optimal solution for Problem (9).

One way to set $\boldsymbol{u}_{l, i}$ is to let $\boldsymbol{u}_{l, i}=\hat{\boldsymbol{g}}_{l, i} /\left\|\hat{\boldsymbol{g}}_{l, i}\right\|$, where $\hat{\boldsymbol{g}}_{l i}$ is the estimation of channel $\boldsymbol{g}_{l, i}$ or one can let it be the eigenvector corresponding to the largest eigenvalue of the matrix $\mathbb{E}_{g}\left[\boldsymbol{g}_{l, i} \boldsymbol{g}_{l, i}^{H}\right]$. This can be considered as the charger $l$ is serving node $i$ by beamforming vector $u_{l, i}$. We use such beamforming vectors as the predetermined beamforming vectors in the simulations.

\section{NUMERICAL SimUlations}

In this section, we evaluate the monitoring performance of the WSN with the optimal energy beamforming (by Algorithm 1), the optimal predetermined energy beamforming (see Section IV.B), and the case not using beamforming (omnidirectional WET). The simulation is done based on Matlab.

In the WSN, we assume that $N$ sensor nodes are randomly deployed in a region of size 30 meters by 30 meters, and a sink is deployed at the center to collect data. The transmission power $e_{i j}^{O}$ for $v_{i}$ to transmit data to $v_{j}$ with distance $d$ is $10^{-7} d^{2}$ Watts, with data rate $250 \mathrm{kbps}$. The energy conversion efficiency $\eta$ is 0.01 . For the charger, its transmission power in WET is 1 Watts, and it has $M$ antennas. The wavelength is 0.01 meter. The channel model of energy transmission is considered as Rician fading model as [21], and is described as $\boldsymbol{g}_{l, i}=\sqrt{\beta K /(K+1)} \boldsymbol{g}_{l, i}^{d}+\sqrt{\beta /(K+1)} \boldsymbol{g}_{l, i}^{s}$, where $\boldsymbol{g}_{l, i}^{d}$ is 
TABLE II: Parameters in the simulations

\begin{tabular}{|c|l|}
\hline symbols & value \\
\hline area size & $30 \mathrm{~m} \times 30 \mathrm{~m}$ \\
\hline data rate & $250 \mathrm{kbps}$ \\
\hline$e_{i j}^{O}$ & $10^{-7} d^{2}$ Watts \\
\hline$P_{l}$ & $1 \mathrm{Watts}$ \\
\hline wavelength of energy beams & $0.01 \mathrm{~m}$ \\
\hline$\eta$ & $1 \%$ \\
\hline
\end{tabular}

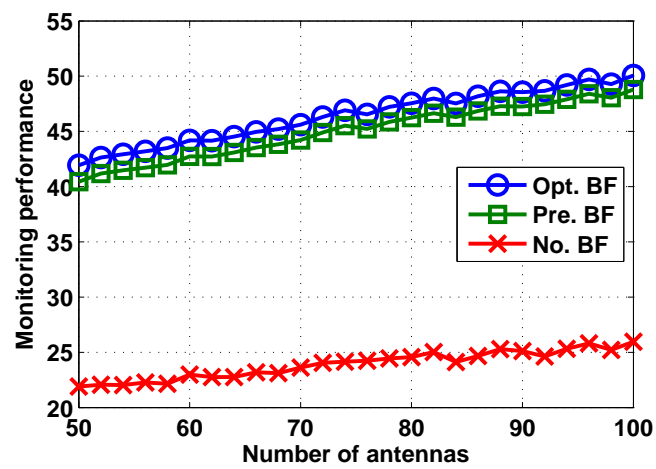

Fig. 2: Comparison of the monitoring performance $\left(\sum_{i} \ln (1+\right.$ $\left.w_{i}\right)$ ) with different numbers of antenna achieved by optimal energy beamforming, predetermined energy beamforming, and no beamforming

a deterministic vector representing the line-of-sight path from charger $l$ to node $i, \boldsymbol{g}_{l, i}^{s} \sim \mathcal{C N}(0, \boldsymbol{I})$ is the scatter component, $\beta$ denotes the path loss, $K$ is the Rician factor, which is set to be 100 in the simulation. Then, $\boldsymbol{K}_{l, i}=\beta K \boldsymbol{g}_{l, i}^{d} \boldsymbol{g}_{l, i}^{d H} /(K+$ $1)+\beta \boldsymbol{I} /(K+1)$. The monitoring performance is defined as $F(\boldsymbol{w})=\sum_{i} \ln \left(1+w_{i}\right)$. The parameters are summarized in Table II.

To begin with, we consider the case with only one charger that transmits energy, and the charger is also the sink that collects data. We fix the number of sensor nodes to be 15 and vary the number of antenna that the charger has from 50 to 100. The result is as shown in Fig. 2. The blue line with circles represents the case of the optimal energy beamforming achieved by Algorithm 1. The green line with squares, and the red line with crosses represents the case of the predetermined energy beamforming, and the case of non beamforming, respectively. It shows that, when the number of antenna increases, the monitoring performances of all schemes increase. The reason is that, with more antennas, the charger can form a sharper beam. Thus, more energy can be received by the sensor nodes. It also shows that, the performances of using beamforming are much better than that of the case without beamforming. It shows the benefits of using beamforming instead of broadcasting the energy. Besides, the performance of the optimal beamforming achieved by Algorithm 1 is slightly better than that of the pre-determined beamforming by solving Problem (9), where the pre-determined beamforming vectors are $\boldsymbol{u}_{l, i}=$ $\hat{\boldsymbol{g}}_{l, i} /\left\|\hat{\boldsymbol{g}}_{l, i}\right\|$. Recall that Problem (9) can be turned into a convex optimization problem where all the constraints are linear, where the optimal beamforming requires solving an

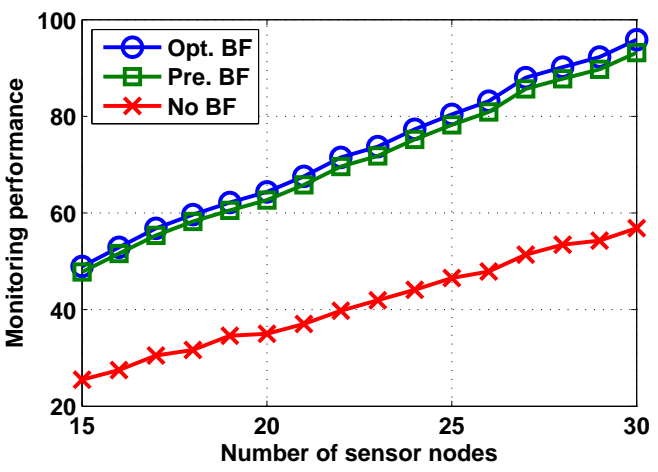

Fig. 3: Comparison of the monitoring performance $\left(\sum_{i} \ln (1+\right.$ $\left.w_{i}\right)$ ) with different numbers of sensor nodes achieved by optimal energy beamforming, predetermined energy beamforming, and no beamforming

SDP, the time complexity for the pre-determined beamforming is much lower than that of the optimal beamforming. Thus, pre-determined beamforming is a good approximation for the optimal beamforming problem.

Then, we fix the number of antennas of the charger to be 100 , and change the number of sensor nodes from 15 to 30. The simulation results are shown in Fig. 3. In general, it shows that, the monitoring performance of using energy beamforming is still much better than the case of WET by broadcasting. Predetermined energy beamfroming is slightly worse than the optimal energy beamforming, which is similar to the case where we vary the number of antenna of the charger. Besides, the monitoring performances of all three schemes increase with the number of sensor nodes. The reason is that, if we deploy a node just act as a pure relay (its sampling rate $w_{i}$ is 0 ), it helps to reduce energy consumptions in data transmission whereas not degenerate the monitoring performance. If we check the average monitoring performance per node $(F(\boldsymbol{w}) / N)$, we can see that the trend is decreasing with the number of sensor nodes but may go up a little sometimes, as shown in Fig. 4. The reason is that, with more sensor nodes, the energy received by each node in average decreases, whilst the energy consumption of a node may also reduce due to the decrease in the average data transmission distance.

We also test the case with multiple chargers. The setting is similar to the case with single charger. The difference is that we have four chargers at $(30,0),(0,30),(-30,0)$, and $(0,-30)$. Each charger has power limit $P_{l}=1.2$ Watts, where the total power limit $P=4$ Watts. The result, as shown in Fig. 5, is similar to the case with a single charger.

To summarize, the monitoring performance improves significantly by using optimal energy beamforming compare to without using energy beamforming. Also, the performance of the predetermined energy beamforming is slightly worse than the optimal energy beamforming. 


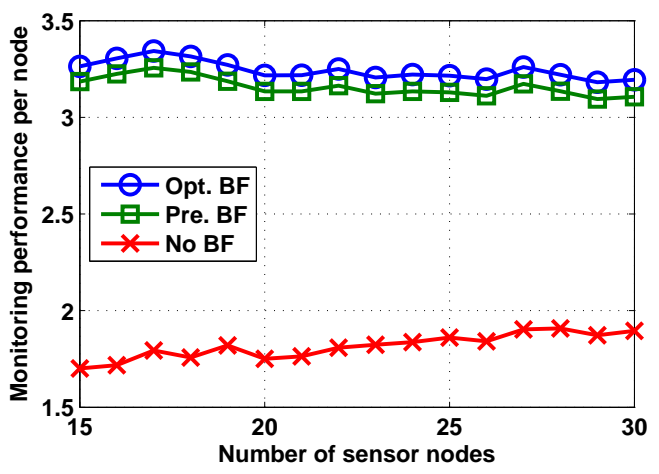

Fig. 4: Comparison of the monitoring performance per node $\left(\sum_{i} \ln \left(1+w_{i}\right) / N\right)$ with different numbers of sensor nodes achieved by optimal energy beamforming, predetermined energy beamforming, and no beamforming

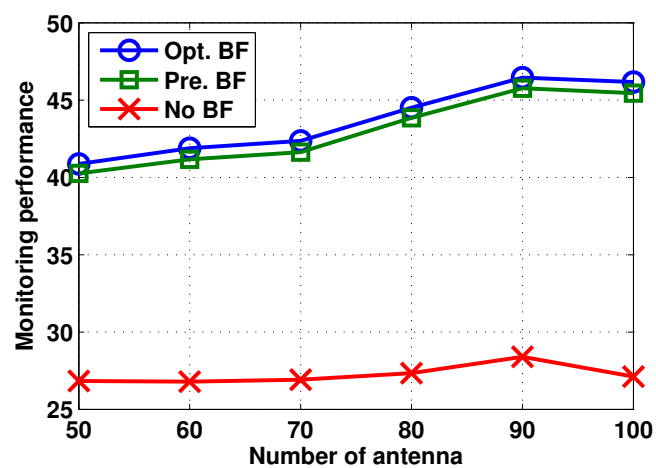

Fig. 5: Comparison of the monitoring performance $\left(\sum_{i} \ln (1+\right.$ $\left.w_{i}\right)$ ) with different numbers of antenna achieved by optimal energy beamforming, predetermined energy beamforming, and no beamforming from four chargers

\section{CONClusions And Future Work}

We have investigated the problem of maximizing the monitoring performance of wireless sensor networks by jointly considering routing and wireless energy transmission. This set-up has led to a non-convex problem formulation. We have transformed the original problem to a semi-definite programming problem, which is solvable, and proved that the optimal solution of the new problem is also the optimal solution of the original problem. Thus, we have proposed an efficient algorithm to solve the original problem. We have also proposed a low-complexity scheme where time sharing between predetermined beam vectors are adopted. The simulation results showed that significant performance gains can be obtained by using optimized energy beamforming over non-optimized energy broadcasting. Besides, the performance of the predetermined energy beamforming scheme is observed to be only slightly worse than the optimal case, which suggests that it could be a good substitute for the optimal beamforming. An interesting future research direction is to consider the scenario where the energy beam vectors have further restrictions, such as the number of different beam vectors that can be employed.

\section{REFERENCES}

[1] L. Mainetti, L. Patrono, and A. Vilei, "Evolution of wireless sensor networks towards the internet of things: A survey," in Proc. IEEE International Conference on Software, Telecommunications and Computer Networks, 2011, pp. 1-6.

[2] S. Sudevalayam and P. Kulkarni, "Energy harvesting sensor nodes: Survey and implications," IEEE Communications Surveys \& Tutorials, vol. 13, no. 3, pp. 443-461, 2011.

[3] S. Ulukus, A. Yener, E. Erkip, O. Simeone, M. Zorzi, P. Grover, and K. Huang, "Energy harvesting wireless communications: A review of recent advances," IEEE Journal on Selected Areas in Communications, vol. 33, no. 3, pp. 360-381, 2015.

[4] O. Galinina, H. Tabassum, K. Mikhaylov, S. Andreev, E. Hossain, and Y. Koucheryavy, "On feasibility of 5g-grade dedicated rf charging technology for wireless-powered wearables," IEEE Wireless Communications, vol. 23, no. 2, pp. 28-37, 2016.

[5] X. Lu, P. Wang, D. Niyato, D. I. Kim, and Z. Han, "Wireless charging technologies: Fundamentals, standards, and network applications," IEEE Communications Surveys \& Tutorials, vol. 18, no. 2, pp. 1413-1452, 2016.

[6] L. Liu, R. Zhang, and K.-C. Chua, "Multi-antenna wireless powered communication with energy beamforming," IEEE Transactions on Communications, vol. 62, no. 12, pp. 4349-4361, 2014.

[7] J. Yang, X. Wu, and J. Wu, "Optimal scheduling of collaborative sensing in energy harvesting sensor networks," IEEE Journal on Selected Areas in Communications, vol. 33, no. 3, pp. 512-523, 2015.

[8] N. Michelusi and M. Zorzi, "Optimal adaptive random multiaccess in energy harvesting wireless sensor networks," IEEE Trans. on Communications, vol. 63, no. 4, pp. 1355-1372, 2015.

[9] L. Xie, Y. Shi, Y. T. Hou, and A. Lou, "Wireless power transfer and applications to sensor networks," IEEE Wireless Communications, vol. 20, no. 4, pp. 140-145, 2013.

[10] G. Yang, C. K. Ho, R. Zhang, and Y. L. Guan, "Throughput optimization for massive mimo systems powered by wireless energy transfer," IEEE Journal on Selected Areas in Communications, vol. 33, no. 8, pp. 1640$1650,2015$.

[11] G. Liu, Z. Wang, and T. Jiang, "Qos-aware throughput maximization in wireless powered underground sensor networks," IEEE Transactions on Communications, vol. 99, no. 99, pp. 1-13, 2016.

[12] R. Du, C. Fischione, and M. Xiao, "Lifetime maximization for sensor networks with wireless energy transfer," in Proc. IEEE International Conference on Communications, 2016, pp. 20-25.

[13] L. Xie, Y. Shi, Y. T. Hou, and H. D. Sherali, "Making sensor networks immortal: An energy-renewal approach with wireless power transfer," IEEE/ACM Trans. on Networking, vol. 20, no. 6, pp. 1748-1761, 2012.

[14] L. Xie, Y. Shi, Y. Hou, W. Lou, H. Sherali, H. Zhou, and S. Midkiff, "A mobile platform for wireless charging and data collection in sensor networks," IEEE Journal on Selected Areas in Communications, vol. 33 no. 8, pp. 1521-1533, Aug 2015.

[15] J.-H. Chang and L. Tassiulas, "Maximum lifetime routing in wireless sensor networks," IEEE/ACM Trans. on Networking, vol. 12, no. 4, pp. 609-619, 2004.

[16] I. Koutsopoulos and M. Halkidi, "Distributed energy-efficient estimation in spatially correlated wireless sensor networks," Computer Communications, vol. 45, pp. 47-58, 2014.

[17] A. Sunny and J. Kuri, "A framework for designing multihop energy harvesting sensor networks," IEEE Journal on Selected Areas in Communications, vol. 34, no. 5, pp. 1491-1501, 2016.

[18] S. Guo, C. Wang, and Y. Yang, "Joint mobile data gathering and energy provisioning in wireless rechargeable sensor networks," IEEE Transactions on Mobile Computing, vol. 13, no. 12, pp. 2836-2852, 2014.

[19] C. Helmberg, F. Rendl, R. J. Vanderbei, and H. Wolkowicz, "An interior-point method for semidefinite programming," SIAM Journal on Optimization, vol. 6, no. 2, pp. 342-361, 1996.

[20] H. Wolkowicz, R. Saigal, and L. Vandenberghe, Handbook of semidefinite programming: theory, algorithms, and applications. Springer Science \& Business Media, 2012, vol. 27.

[21] S. Kashyap, E. Björnson, and E. G. Larsson, "On the feasibility of wireless energy transfer using massive antenna arrays," IEEE Transactions on Wireless Communications, vol. 15, no. 5, pp. 34663480, 2016. 